\title{
Theoretical-experimental method of multiscale modelling of two-component materials
}

\author{
Anguel Baltov ${ }^{1, *}$, Ana Yanakieva ${ }^{1}$ \\ ${ }^{1}$ Institute of Mechanics, Bulgarian Academy of Sciences, 1113 Sofia, 4 G.Bonchev str., Bulgaria
}

\begin{abstract}
Modern mechano-mathematical models of materials are multilevel ones combining macro-, meso- and micro-level models. A major problem however is how to present a clear link between the levels. Moreover, structural models should be well verified, reflecting material behavior at meso-level and its microstructure. Hence, we propose a theoretical-experimental approach to multilevel modeling, treating discs which operate under plane stress. The approach comprises the following stages: I stage: Identify a system of characteristic points on the disc surface. Employing an appropriate optical method, find point displacements using time steps in the course of deformation. II stage: Based on displacement development in time, rationally find the variation of the strain tensor components using strains directed from the main point to the closest points of its vicinity. III stage: Define a meso-representative element in the disc plane as an ellipse with diameters related to the principal strains. IV stage: Having prepared metallographic micro-sections, define systems of characteristic structural elements in the mesorepresentative ellipses. V stage: Averaging the structural systems at mesolevel, define macro-parameters involving material deformation parameters. VI stage: Find material macro-deformation parameters on the basis of an appropriate design of micro-representative elements and calculate the stressed-strained state of the latter applying representative loading. A test example is give in order to illustrate the approach.
\end{abstract}

\section{Introduction}

A modern tendency in the mechanics of materials is the design of mechano-mathematical models accounting for the material structure at three scales: micro-, meso- and macro-ones $[1,2]$. The arising major problems of multiscale modeling are: (1) Design of a particular model with specific parameters and development of a method of parameter identification at each scale; (2) Identification of the link between respective models and parameters, as well as verification of scale transition.

Multi-scale models with their plausibility and reliability are especially useful in modeling and calculation of safe structures and as a result- in saving energy needed for structure erection and exploitation. We focus in what follows on the analysis of materials with grain structure consisting of two components: a matrix and grain inclusions, which

\footnotetext{
*Corresponding author: anguelbaltov@gmail.com
} 
generally reinforce the material. Models at all the three scales are designed within the frames of continuum mechanics. Such an approach seems reasonable enough, since we do not analyse sub micro-scale of a material with a discrete atomic or molecular structure. In view of a possible application of some experimental methods we consider thin disks under plane stress undergoing static external loading. Disc thickness is much smaller than that of the grains. We treat disks as structural elements operating in exploitation conditions, i.e. they deform purely elastically without the development of damage and inelastic strains. The elastic properties are assumed to be isotropic and non-homogeneous, and they may change as a result of eventual structural changes occurring at meso- level.

The paper offers a theoretical-experimental method of multi-scale modelling of the materials concerned. It is based on generalization and further elaboration of a number of results published in refs. [3-6], adopting stages of an eventual practical realization.

\section{First stage: Macro-experiments on disks}

Consider thin elastically deformed disks undergoing static loading which generally varies in time $t \in\left[t_{0}, t_{f}\right]$. Select $n$ characteristic points on the disk surface with projections on the disk middle surface $-M(p, t)\left\{\begin{array}{l}X_{M}(p, t) \\ Y_{M}(p, t)\end{array}\right\}, p=1,2 \ldots n$ where a coordinate system $O X Y Z$ is introduced, plane $O X Y$ coincides with the disk middle surface and axis $O Z$ is directed along the disk thickness, Fig. 1.



Fig. 1. A thin disk with characteristic points on its middle surface

Consider each point $M(p, t)$ together with the close characteristic points $N(p, j, t)$, $j=1,2 \ldots s$ that bound it. Discretize the process in time $t_{k}, k=0,1,2 \ldots m$, where $t_{0}$ is process start and $t_{f}=t_{m}$ is process finish. Adopt identical intervals $\Delta t=t_{k}-t_{k-1}$ for each $k$. Then, one can find the location of points $M\left(p, t_{k}\right)$ (respectively $N\left(p, j, t_{k}\right)$ ) at 
each time $t_{k}$ (this can be done using different optical methods to locate the point position [11]) and specify vector $\overrightarrow{M N}$ with magnitude

$$
|M N|=C\left(p, j, t_{k}\right)=\sqrt{\left[X_{N}\left(p, j, t_{k}\right)-X_{M}\left(p, t_{k}\right)\right]^{2}+\left[Y_{N}\left(p, j, t_{k}\right)-Y_{M}\left(p, t_{K}\right)\right]}
$$

The respective unit vector is

$$
\vec{n}\left(p, j, t_{k}\right)=\left\{\begin{array}{c}
n_{X}\left(p, j, t_{k}\right)=\frac{X_{N}\left(p, j, t_{k}\right)-X_{M}\left(p, t_{k}\right)}{C\left(p, j, t_{k}\right)} \\
n_{Y}\left(p, j, t_{k}\right)=\frac{Y_{N}\left(p, j, t_{k}\right)-Y_{M}\left(p, t_{k}\right)}{C\left(p, j, t_{k}\right)} \\
p=1,2 \ldots n ; j=1,2 \ldots s ; k=0,1,2 \ldots m
\end{array}\right.
$$

We determine approximately strains directed along $\vec{n}\left(p, j, t_{k}\right)$ using the selected characteristic points and the calculated distances $|M N|=C\left(p, j, t_{k}\right)$ for each $p, j$ and $k$

$$
E_{n}^{\exp }\left(p, j, t_{k}\right)=\frac{C\left(p, j, t_{k}\right)-\bar{C}\left(p, j, t_{k-1}\right)}{\bar{C}\left(p, j, t_{k-1}\right)}
$$

where $\bar{C}\left(p, j, t_{k}\right)$ is the magnitude of $\overrightarrow{M N}\left(p, j, t_{k-1}\right)$, the vector rotating as an ideal solid.

The experimentally found strain $E_{n}^{\exp }\left(p, j, t_{k}\right)$ is compared to the theoretically calculated one $E_{n}^{\text {th }}\left(p, j, t_{k}\right)$, using a known formula [9]

$$
\begin{array}{r}
E_{n}^{t h}\left(p, j, t_{k}\right)=E_{X X}\left(p, t_{k}\right)\left[n_{X}\left(p, j, t_{k}\right)\right]^{2}+E_{Y Y}\left(p, t_{k}\right)\left[n_{Y}\left(p, j, t_{k}\right)\right]^{2}+ \\
+E_{X Y}\left(p, t_{k}\right)\left[n_{X}\left(p, j, t_{k}\right) n_{Y}\left(p, j, t_{k}\right)\right]
\end{array}
$$

Design functional $L\left(p, t_{k}\right)$ of the least square deviation between the experimental (eq. 3) and the theoretical strains (eq. 4).

$$
L\left(p, t_{k}\right)=\sqrt{\frac{\sum_{j=1}^{S}\left[E_{n}^{\exp }\left(p, j, t_{k}\right)-E_{n}^{t h}\left(p, j, t_{k}\right)\right]^{2}}{S-1}}
$$


Using condition for $L_{\text {min }}$ we find the components of the strain tensor $E_{X X}\left(p, t_{k}\right), E_{Y Y}\left(p, t_{k}\right)$ and $E_{X Y}\left(p, t_{k}\right)$ at point $M\left(p, t_{k}\right)$ and time $t_{k}$

$$
\frac{\partial L}{\partial\left[E_{X X}\left(p, t_{k}\right)\right]}=0, \quad \frac{\partial L}{\partial\left[E_{Y Y}\left(p, t_{k}\right)\right]}=0, \quad \frac{\partial L}{\partial\left[E_{X Y}\left(p, t_{k}\right)\right]}=0
$$

To find transversal strain $E_{Z Z}\left(p, t_{k}\right)$ we should measure the change of the thickness $\delta\left(p, t_{k}\right)$ at point $M\left(p, t_{k}\right)$ and time $t_{k}$, i.e.

$$
E_{Z Z}\left(p, t_{k}\right)=\frac{\delta\left(p, t_{k}\right)-\delta\left(p, t_{k-1}\right)}{\delta\left(p, t_{k-1}\right)}
$$

We can calculate the increment $\Delta E_{Z Z}\left(p, t_{k}\right)$ in the interval $\Delta t$ through (7). On the other hand, the increments of $E_{X X}\left(p, t_{k}\right), E_{Y Y}\left(p, t_{k}\right)$ and $E_{X Y}\left(p, t_{k}\right)$ are found through a system of equations involving two times $-t_{k-1}$ and $t_{k}, k=0,1,2 \ldots m$.

Following a method described in [5] we can find the stress tensors with components $\Sigma_{X X}\left(p, t_{k}\right), \Sigma_{Y Y}\left(p, t_{k}\right)$ and $\Sigma_{X Y}\left(p, t_{k}\right)$, and the respective increments $\Delta \Sigma_{X X}\left(p, t_{k}\right)$, $\Delta \Sigma_{Y Y}\left(p, t_{k}\right)$ and $\Delta \Sigma_{X Y}\left(p, t_{k}\right)$. We apply our methodology starting from points close to the boundary of the disk, whereas some points $N\left(p, j, t_{k}\right)$ lie on that boundary. Then, using Hooke's law, we can also find the elasticity characteristics $E_{\text {mac }}\left(p, t_{k}\right), v_{\text {mac }}\left(p, t_{k}\right)$ and $\Delta E_{m a c}\left(p, t_{k}\right), \Delta v_{m a c}\left(p, t_{k}\right)$, respectively.

If the material is homogeneous within the frames of the statistical error and there are no structural changes, the elasticity characteristics will be constant- $E_{\text {mac }}=$ const and $v_{\text {mac }}=$ const . Then, it is easy to obtain the macro elastic characteristics using a different experimental technique [10].

\section{Second stage: Specification of a meso-representative element and meso-structural parameters}

We found the components of the macro tensor at selected characteristic points $\tilde{M}\left(p, t_{k}\right)$ and at each discrete time $t_{k}$. Hence, we can also find the principal strains in the disk middle plane, $E_{N}\left(p, t_{k}\right),(N=I, I I)$. We design meso-representative elements at point $\tilde{M}\left(p, t_{k}\right)$. They are ellipses with diameter $D_{N}=1+E_{N}\left(p, t_{k}\right)$, with axes directed along the principal strains and concluding angle $\alpha_{M}\left(p, t_{k}\right)$ with OX. The height of an elliptic cylinder thus formed is $\delta\left(p, t_{k}\right)$, whose section cut by the middle plane is an ellipse, Fig. 2.

Considering each representative element at point $\tilde{M}\left(p, t_{k}\right)$, we grind the surface around the representative ellipse, which is a projection of the elliptical cylinder. Using an electron microscope, we identify the grain inclusions. Each grain is approximated as an 
elliptic cylinder, whose section cut by the middles plane is an ellipse with point $Q\left(p, t_{k} ; q\right)$ as a center. Moreover, its diameters are $d_{1}\left(p, t_{k} ; q\right)$ and $d_{2}\left(p, t_{k} ; q\right)$, its height is $\delta\left(p, t_{k}\right), q$ is the consecutive grain number, $q=1,2 \ldots q_{n}, q_{n}$ is the general number of grains within the representative meso-element at $\tilde{M}\left(p, t_{k}\right)$.



Fig. 2. A meso-representative element. Approximated element grains

Consider the middle plane of the cross section of the meso-representative element. Introduce a coordinate system $\tilde{M} x y$ with meso-strains $\varepsilon_{x x}\left(p, t_{k}\right), \varepsilon_{y y}\left(p, t_{k}\right)$ and $\varepsilon_{x y}\left(p, t_{k}\right)$ and meso-stresses $\sigma_{x x}\left(p, t_{k}\right), \sigma_{y y}\left(p, t_{k}\right)$ and $\sigma_{x y}\left(p, t_{k}\right)$. Introduce into each grain a local coordinate system $Q \xi \eta$ with micro-strains $e_{\xi \xi}\left(p, t_{k} ; q\right), e_{\eta \eta}\left(p, t_{k} ; q\right)$ and $e_{\xi \eta}\left(p, t_{k} ; q\right)$ and micro-stresses $s_{\xi \xi}\left(p, t_{k} ; q\right), s_{\eta \eta}\left(p, t_{k} ; q\right)$ and $s_{\xi \eta}\left(p, t_{k} ; q\right)$. Axis $O X$ and line $\tilde{M} x$ conclude angle $\alpha_{M}\left(p, t_{k}\right)$, while $\tilde{M} x$ and $Q \xi$ conclude angle $\beta_{Q}\left(p, t_{k} ; q\right)$.

Divide the grains of the meso-representative element at point $\tilde{M}\left(p, t_{k}\right)$ into grain systems (a) with identical slope $\beta_{Q}(a), a=1,2 \ldots n_{a}$, where $n_{a}$ is the number of grain systems in that element. Each system (a) has $r_{a}$ number of grains where $\sum_{a=1}^{n_{a}} r_{a}=n_{q}$ is the total number of grains in (a) system. Each grain has different diameters $d_{1}\left(g_{a}\right), d_{2}\left(g_{a}\right)$ and the distance $l\left(g_{a}\right)$ between it and the neighboring grains of every system (a) is minimal. We calculated for system (a) the mean values $\bar{d}_{1}(a), \bar{d}_{2}(a)$ and $\bar{l}(a)$, Fig. 3 . 


\section{Third stage: Identification of the structural parameters at the three scales}

\subsection{Micro-scale structural parameters}

We select a micro-structural element for system (a): a prism with rectangular cross section $\bar{l}(a) \times \bar{l}(a)$ in the disk middle plane. Its center is $Q_{a}\left(p, t_{k}\right) \subset Q\left(p, t_{k} ; q\right)$ located in the middle of the representative inclusion- an ellipse with diameters $\bar{d}_{1}(a), \bar{d}_{2}(a)$. Its height is $\delta\left(p, t_{k}\right)$, being one and the same for the entire meso-representative element at point $\tilde{M}\left(p, t_{k}\right)$, Fig. 3 .

The micro-representative element at point $Q_{a}\left(p, t_{k}\right)$ is loaded conditionally by a uniformly distributed load applied along the element border- see the representative loading in [5]. This loading yields uniformly distributed meso-stresses at $Q_{a}\left(p, t_{k}\right)$, according to the scheme in [5].

Using nanoindentation [9] we find material micro-elasticity characteristics: $E_{\text {mic }}$ (mat), $v_{\text {mic }}$ (mat) of the matrix and $E_{\text {mic }}(\mathrm{incl}), v_{\text {mic }}(\mathrm{incl})$ of the inclusions. Micro-stresses and micro-strains at point $Q_{a}\left(p, t_{k}\right)$ are determined via FEM [12]. Micro-strains are presented as $e_{\xi \xi}\left( \pm \frac{\bar{l}(a)}{2}, \eta, t_{k}\right), e_{\eta \eta}\left(\xi, \pm \frac{\bar{l}(a)}{2}, t_{k}\right), e_{\xi \eta}\left( \pm \frac{\bar{l}(a)}{2}, \eta, t_{k}\right)$ at the element border, where $\xi=\eta= \pm \frac{\bar{l}(a)}{2}$.

Thus, meso-strains at point $Q_{a}\left(p, t_{k}\right)$ are found, performing averaging according to the following expressions:

$$
\begin{aligned}
\varepsilon_{\xi \xi}\left(p, t_{k} ; a\right) & =\int_{-\bar{l}(a) / 2}^{\bar{l}(a) / 2} e_{\xi \xi}\left(\frac{\bar{l}(a)}{2}, \eta, t_{k}\right) d \eta \\
\varepsilon_{\eta \eta}\left(p, t_{k} ; a\right) & =\int_{-\bar{l}(a) / 2}^{\bar{l}(a) / 2} e_{\eta \eta}\left(\frac{\bar{l}(a)}{2}, \eta, t_{k}\right) d \eta \\
\varepsilon_{\xi \eta}\left(p, t_{k} ; a\right) & =\int_{-\bar{l}(a) / 2}^{\bar{l}(a) / 2} e_{\xi \eta}\left(\frac{\bar{l}(a)}{2}, \eta, t_{k}\right) d \eta
\end{aligned}
$$




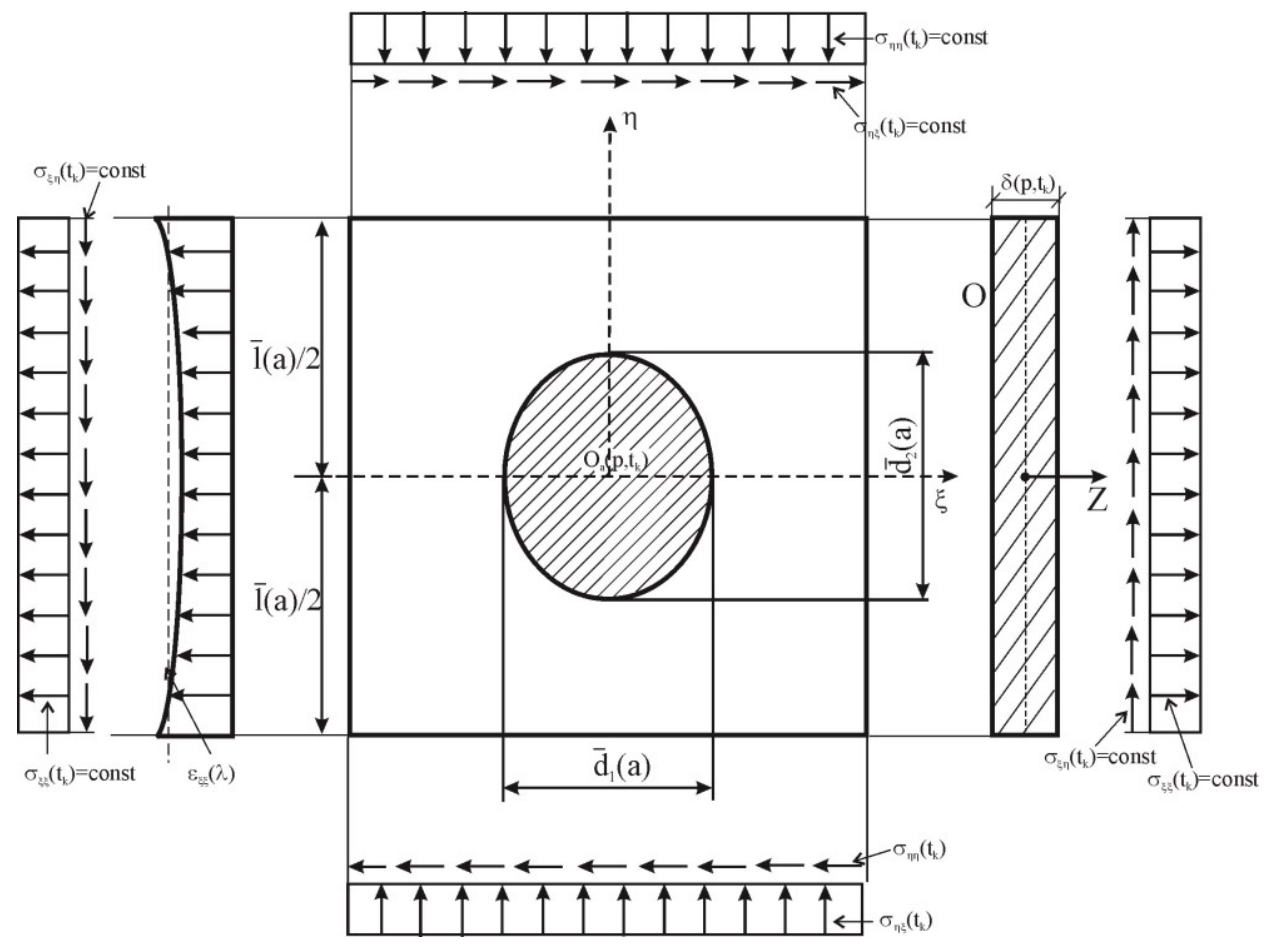

Fig. 3. Micro-representative element and essential constant loading by meso-stresses

Meso-scale stresses and strains at point $Q_{a}\left(p, t_{k}\right)$ are linked to each other via Hooke's law, using the meso-elastic characteristics $E_{\text {mes }}(a)$ and $v_{\text {mes }}(a)$. We have for system (a) that

$$
\begin{aligned}
& \varepsilon_{\xi \xi}\left(p, t_{k} ; a\right)=\frac{1}{E_{\text {mes }}(a)}\left[\sigma_{\xi \xi}\left(p, t_{k} ; a\right)-v_{\text {mes }}(a) \sigma_{\eta \eta}\left(p, t_{k} ; a\right)\right] \\
& \varepsilon_{\eta \eta}\left(p, t_{k} ; a\right)=\frac{1}{E_{\text {mes }}(a)}\left[\sigma_{\eta \eta}\left(p, t_{k} ; a\right)-v_{\text {mes }}(a) \sigma_{\xi \xi}\left(p, t_{k} ; a\right)\right]
\end{aligned}
$$

Using system (9) we find those meso-elastic characteristics, assuming that they are valid for all systems (a). If we want to obtain the real meso-stresses at the point $M\left(p, t_{k}\right)$, the meso-ellipse can be enveloped by a rectangle, Fig 2 . Then, the new rectangular element can be uniformly loaded by $\Sigma_{X X}\left(p, t_{k}\right), \Sigma_{Y Y}\left(p, t_{k}\right)$ and $\Sigma_{X Y}\left(p, t_{k}\right)$, according to the scheme in [5], Fig. 3, and the calculations can be performed using FEM [12].

The following parameter characterizes the meso-structure

$$
K_{a}=\frac{E_{\text {mes }}^{\prime}(a)}{E_{\text {mac }}^{\prime}} ; \quad E_{\text {mes }}^{\prime}(a)=\frac{E_{\text {mes }}(a)}{1-v_{\text {mes }}(a)}, \quad E_{\text {mac }}^{\prime}=\frac{E_{\text {mac }}}{1-v_{\text {mac }}}
$$


It presents the effect of the microstructure (divided into grain systems) on the model parameters at the meso-scale.

\subsection{Meso-scale structural parameters}

We introduce a meso-scale structural parameter of the grain system (a) as a vector having the form

$$
J_{\zeta}(a)=m_{\zeta}(a) \varphi_{a}\left(p, t_{k}\right) r_{a} K_{a}\left(p, t_{k}\right), \quad \zeta=\xi, \eta
$$

where $m_{\zeta}(a)$ gives the grain orientation of the system (a) e.g.

$$
m_{\zeta}(a),\left\{\begin{array}{l}
m_{\xi}(a)=\cos \beta_{Q}(a) \\
m_{\eta}(a)=\sin \beta_{Q}(a)
\end{array}\right.
$$

We have $\varphi_{a}\left(p, t_{k}\right)=\frac{\bar{F}_{\text {mic }}(a)}{\bar{F}_{e l}\left(p, t_{k}\right)}, \quad \bar{F}_{m i c}(a)=\pi \bar{d}_{M}(a), \quad \bar{d}_{M}(a)=0,5\left(\bar{d}_{1}(a)+\bar{d}_{2}(a)\right)$ and $\bar{F}_{e l}\left(p, t_{k}\right)=\pi d_{M}^{2}\left(p, t_{k}\right), d_{M}=\frac{1}{2}\left(d_{I}\left(p, t_{k}\right)+d_{I I}\left(p, t_{k}\right)\right)$, Fig. 2, which gives the effect of the grain size on different systems (a). The mechanical coefficient $K_{a}$ is found using expression (10).

Based on $J_{\zeta}(a)$ we introduce a meso-structural parameter $I_{\zeta}\left(p, t_{k}\right)$ which expresses the prevailing grain orientation:

$$
I_{\zeta}\left(p, t_{k}\right)=\sum_{a=1}^{n_{a}} J_{\zeta}(a), \zeta=\xi, \eta
$$

It unifies all systems (a) within the meso-representative element at point $\tilde{M}\left(p, t_{k}\right)$.

\subsection{Macro-scale structural parameters}

Based on the vector parameter $I_{\xi}\left(p, t_{k}\right)$ we introduce its magnitude as a macro scale structural parameter

$$
I_{0}\left(p, t_{k}\right)=\sqrt{I_{\xi}^{2}\left(p, t_{k}\right)+I_{\eta}^{2}\left(p, t_{k}\right)}
$$

The macro elasticity characteristics $E_{\text {mac }}$ and $v_{\text {mac }}$ depend on $I_{0}\left(p, t_{k}\right)$. If real structures presented at macro-scale by different parameters $I_{0}\left(p, t_{k}\right)$ are considered, the dependence can be found theoretically [32] determining the macro elasticity characteristics $E_{\text {mac }}\left(p, t_{k}\right)=\Phi\left(I_{0}\left(p, t_{k}\right)\right)$ and $v_{\text {mac }}\left(p, t_{k}\right)=\Psi\left(I_{0}\left(p, t_{k}\right)\right)$ where $\Phi$ and $\Psi$ are approximation functions. For elastically deforming bodies that keep their structure, we have $I_{0}=I_{0}(p)$. If the material is homogeneous $I_{0}$ does not depend on $p$. So far we identified the scale structural parameters. Note that energetic links exist between the three scales (at point $\tilde{M}\left(p, t_{k}\right)$ ) which have been discussed in [4]: 


$$
\Sigma_{I J}\left(p, t_{k}\right) E_{I J}\left(p, t_{k}\right)=\int_{F_{e l}} \sigma_{i j}\left(p, t_{k}\right) \varepsilon_{i j}\left(p, t_{k}\right) d F,(I, J=X, Y ; i, j=x, y)
$$

Table 1. Grain systems.

\begin{tabular}{|c|c|c|r|r|r|r|r|r|}
\hline$a$ & $\beta_{a}(a)$ & $r_{a}$ & $\bar{d}_{1}(a)[\mu m]$ & $\bar{d}_{2}(a)[\mu m]$ & $F_{\text {incl }}(a)\left[\mu m^{2}\right]$ & $\varphi_{a}$ & $K_{a}$ & $\begin{array}{r}J_{\xi}(a) \\
J_{\eta}(a)\end{array}$ \\
\hline 1 & $0^{0}$ & 15 & 0,224 & 0,101 & 0,0834 & 0,0033 & 0,86 & $\begin{array}{r}0,0043 \\
0,0000\end{array}$ \\
\hline 2 & $15^{0}$ & 3 & 0,205 & 0,128 & 0,0876 & 0,0035 & 0,87 & $\begin{array}{r}0,0088 \\
0,0024\end{array}$ \\
\hline 3 & $30^{0}$ & 2 & 0,285 & 0,205 & 0,1900 & 0,0076 & 0,90 & $\begin{array}{r}0,0012 \\
0,0007\end{array}$ \\
\hline 4 & $45^{0}$ & 3 & 0,137 & 0,082 & 0,0380 & 0,0151 & 0,95 & $\begin{array}{r}0,0030 \\
0,0030\end{array}$ \\
\hline 5 & $60^{0}$ & 2 & 0,225 & 0,090 & 0,0780 & 0,0031 & 0,85 & $\begin{array}{r}0,0029 \\
0,0051\end{array}$ \\
\hline 6 & $120^{0}$ & 6 & 0,167 & 0,081 & 0,0490 & 0,0120 & 0,92 & $\begin{array}{r}-0,0055 \\
0,0095\end{array}$ \\
\hline 7 & $150^{0}$ & 12 & 0,206 & 0,101 & 0,0740 & 0,0030 & 0,84 & $\begin{array}{r}-0,0261 \\
0,0151\end{array}$ \\
\hline 8 & $165^{0}$ & 4 & 0,363 & 0,148 & 0,2042 & 0,0032 & 0,91 & $\begin{array}{r}-0,0287 \\
0,0077\end{array}$ \\
\hline
\end{tabular}

\section{Test example}

We use the method proposed to illustrate the efficiency of Stage 3 and Stage 4, mainly, treating a two-component nanocomposite with a matrix consisting of polypropylene and polymer glue and reinforced by means of grains composed of carbon tube bundles. The material was analyzed in detail in [7]. We use a representative microstructure pattern found by preparing a metallographic micro-section and magnifying it via an electron microscope type SEM. No damage and change of the structure are observed in the range of the elastic strains.

For example we consider a meso-structural element with 47 grains $\left(\eta_{q}=47\right)$ distributed within eight grain system (a) $\left(n_{a}=8\right)$. The accuracy of $\beta_{Q}(a)$ is $2^{0}$, Table 1 . The table presents: systems angles $\beta_{Q}(a)$; number of grains $r_{a}$ in each system (a); mean diameters $\bar{d}_{1}(a)$ and $\bar{d}_{2}(a)$; coefficients $\varphi_{a}$; structural parameters $K_{a}$ and the components of the meso-structural parameters $I_{\xi}(a)$ for all systems (a).

We calculate the following vector measure

$$
I_{\zeta},\left\{\begin{array}{l}
I_{\xi}=-0,0167 \\
I_{\eta}=+0,0435
\end{array}\right.
$$

The macro structural measure is $I_{0}=+0,0466$. 


\section{Conclusions}

The method presented in the study is based on available experimental data and theoretical studies and results. It provides means of the design of physically clear structural parameters at the three scales: macro-, meso- and micro-ones, specifying a link between them. This makes the mechano-mathematical model a physically well-defined tool.

The employment of a multi-scale model in the mechanics of materials is an important issue enabling one to describe adequately material mechanical behavior and explicitly account for the effect of material structure. The model is exceptionally useful in structural mechanics since it allows a plausible record of the behavior of structural elements. It is also useful in modern energy saving structures compounded by surface-reinforced elements [8] providing an appropriate modeling of the reinforcement.

The study has been supported by the National Fund "Scientific Research", Project DFNI E02/10 121214.

\section{References}

1. A. Roos, et al, Int. J of Plastisity, 20, 811-830, (2004)

2. F. D. Ficher, E.Werner, ZAMM, 77, 341-348, (1977)

3. A. Baltov, J. Th. Appl. Mech., 40 (4), 41-54, (2010)

4. A. Baltov, 12th Nat. Congress on Th. and Appl. Mechanics, Varna, (On CD), (2013)

5. A. Baltov, A. Yanakieva, J. of Th. and Appl. Mech., 43 (3), 43-50, (2011)

6. A. Baltov, A. Yanakieva, N. Nikolov, 14th Int. Conf. VSU'2014, 2, 19-25, (2014)

7. Y. Dong, et al, Compos. Sci. Technol., 68, (14), 2864-2875, (2008)

8. A. Baltov, A. Yanakieva, G. Nikolova, Springer Proc. Materials, 3rd International Congress on Energy Efficiency and Energy Related Materials, Oludeniz, Turkey, 167174, (2015)

9. X. Li, B. Bhushan, Materials Characterization, 48, (1), 11-36, (2002)

10. W. Szczepinski, Experimental Methods in Mechanics of Solids, Amsterdam, Elsevier, (1990)

11. V. Kavardzhikov, I. Nikolov, D. Pashkouleva, Series in Applied Mathematics and Mechanics, 5, Institute of Mechanics - BAS, Sofia, (2015)

12. K. Kazakov, Theory of Elasticity, Stability and Dynamics of Structures, $2^{\text {nd }}$ ed., Academic Publishing House of BAS, Sofia, (2011) 